\title{
Nuevas características del trabajador bancario venezolano*
}

\author{
González, María Candelaria** \\ Bracho de Hernández, Yajaira*** \\ Álvarez, Anaís ${ }^{\star * *}$
}

\section{Resumen}

El objetivo del siguiente artículo es identificar las características de las relaciones laborales de la banca universal venezolana, después del proceso de fusiones de 1994. La metodología utilizada fue una revisión bibliográfica y hemerog ráfica para analizar la evolución de las fusiones en el sistema financiero venezolano y la estructura que posee el mismo hasta el 2006 y las incidencias a nivel internacional del proceso de fusiones y posteriormente se realizó un trabajo de campo en Banesco Banco Universal seleccionando una muestra de 9 empleados. Los resultados obtenidos evidencian que existen cambios de tipo numérica, salarial, funcional, cualitativa y de captación y reclutamiento del personal. Se recomienda en futuras investigaciones compararel punto de vista de los empleados con el de la gerencia y con el sindicato; para realizar de esta manera una triada de opiniones para contrastar y verificar la información suministrada por los diferentes actores.

Palabras clave: Fusiones bancarias, relaciones laborales, banca universal, Venezuela, Banesco.

Recibido: 08-02-07. Aceptado: 02-07-07

Resultados preliminares de la Tesis Doctoral "Flexibilización de las Relaciones Laborales en la Banca Universal Venezolana. Periodo 1994-2006.

Resultado del proyecto de investigación registrado ante el CONDES “Modernización Empresarial II Etapa". CONDES No. 0466-2003.

** Magíster en Gerencia de Empresas. Profesor Asociado de la Universidad del Zulia. Investigadora del Centro de Estudios de la Empresa. Facultad de Ciencias Económicas y Sociales. Cursante del doctorado en Ciencias Sociales mención Gerencia. Maracaibo-Venezuela.

E-mail: mcandelag@cantv.net, mcandelag@hotmail.com.

*** Economista, Magíster en Gerencia de Empresas. Profesora Titular e Investigadora de la Universidad del Zulia. Cursante del Doctorado en Ciencias Sociales, Mención Gerencia-LUZ.

E-mail: brachoyajaira@hotmail.com

**** Economista, Magíster en Gerencia de Empresas. Profesora Asociada e Investigadora de la Universidad del Zulia. Cursante del Doctorado en Ciencias Sociales, Mención Gerencia-LUZ.

E-mail: anajalvarez@yahoo.com 


\title{
New Characteristics of the Venezuelan Bank Worker
}

\begin{abstract}
The objective of this article is to identify the characteristics of labor relations in the Venezuelan Universal Bank after the 1994 merger process. The methodology used was a bibliographic and hemerographic review analyzing the evolution of mergers in the Venezuelan financial system and its structure up to 2006, and the incidence of mergers on the international level; later, a field study was made at the Banesco Universal Bank, selecting a sample of 9 employees. Results showed the existence of numerical, salary, functional and qualitative changes as well as changes in personnel training and recruiting. It is recommended that future investigations compare the viewpoints of employees with those of management and the labor union in order to obtain a triad of opinions to contrast and verify the information provided by the different actors.
\end{abstract}

Key words: $\quad$ Bank mergers, labor relations, Universal Bank, Venezuela, Banesco.

\section{Introducción}

Dentro de todos los sectores empleadores en Venezuela; el sector financiero ha sufrido grandes cambios y grandes despidos en últimos diez años, por eso es importante analizar su comportamiento. Sin duda alguna las empresas de todos los sectores han implementado un gran número de estrategias ${ }^{1}$ de modernización para así poder adaptarse a los cambios que se están suscitando a nivel mundial.
La evolución en todo el mundo, tanto a nivel macroeconómico como sectorial, se caracteriza en particular por la liberalización, la eliminación de restricciones y la privatización, las cuales están vinculadas a la intensificación de los procesos de mundialización bancario y han generado un aumento de la presión de la competencia, empujando al sector hacia una mayor concentración de las instituciones en la banca global o universal a través de fusiones y adquisiciones. Dicha presión se ha visto intensificada por la desreglamentación ${ }^{2}$ del siste-

1 Las estrategias empresariales son los medios para lograr objetivos de cambio a mediano y largo plazo, para obtener ventajas comparativas u oponerse a las actuaciones presentes o futuras de los competidores. Por consiguiente, a ellas subyacen principios, valores y fines que determinan la dirección de la empresa; además, la estrategia exige un conjunto de esfuerzos para coordinar e integrar en un todo coherente, recursos y operaciones en función de alcanzar fines u objetivos generales... (Gamboa et al., 2001:138-139).

2 La desreglamentación, o desregulación bien caracterizada a nivel mundial por la libre entrada de los capitales foráneos o extranjeros a competir en los sectores financieros nacionales y a la homogenización de los sistemas normativos en todo el mundo con las normas del comité de prácticas bancarias de Basilea. 
ma financiero internacional, la creciente inestabilidad de los flujos financieros, y el gran número de crisis financieras ocurridas en los últimos años.

Por lo general, se acepta que la fase siguiente al proceso de consolidación del sector puede traer consigo fusiones $\mathrm{y}$ adquisiciones nacionales $\mathrm{y} / \mathrm{o}$ transnacionales. Los rápidos cambios tecnológicos actuales, las tendencias en materia de elaboración de productos, los nuevos sistemas de entrega de servicios al cliente, los cambios operados en los canales de distribución y una convergencia con otros sectores, han complicado los desafíos competitivos a los que se enfrentan los operadores de servicios financieros. Esta evolución ha dado lugar a grandes cambios en lo concerniente a la organización del trabajo y las capacidades exigidas, así como en la cantidad, calidad y condiciones del empleo en el sector.

Hay ciertos detalles de tipo laboral que vienen incorporados en los procesos de fusiones de las entidades bancarias, estos detalles dependen de la forma como se realicen las fusiones y cuáles son los bancos involucrados. Por ello surge la necesidad de estudiar los diferentes cambios que se han dado en las relaciones laborales de la banca universal venezolana a partir del proceso de fusiones que se diera como consecuencia de la crisis financiera de 1994.

La metodología empleada se divide en una revisión bibliográfica y hemerográfica para describir y analizar la evolución de las fusiones a nivel internacional y nacional y su incidencia en las nuevas características del trabajador bancario. Posteriormente se aplicó en Banesco Banco Universal un cuestionario a los tra- bajadores que cumplieran con los siguientes criterios de selección: que sobrevivieran al proceso de fusiones de la institución y que tuviesen un nivel académico mínimo de técnico superior universitario (TSU).

Si bien es cierto fue difícil aplicarles el cuestionario a una muestra probabilísticamente calculada dada la limitación de la confidencialidad y acceso a la información. Este fue aplicado a los trabajadores que accedieran a dar la información y que se tuviera acceso a ellos por la ayuda brindada por contactos en las instituciones financieras; además de contar con los criterios de inclusión del estudio; siendo el muestreo utilizado de tipo intencional. De estos trabajadores se seleccionó un total de 9 que estaban clasificados de la siguiente manera: 6 promotoras, 2 gerentes de oficinas y 1 gerente de ventas, siendo de nivel medio o gerencial. Este fue aplicado en diferentes agencias de la zona Occidente específicamente en la ciudad de Maracaibo, en el periodo enero-marzo de 2006. La entrevista consta de 56 preguntas abiertas y cerradas.

Estos resultados son preliminares y corresponden a una prueba piloto que servirá para la corrección del instrumento de recolección de información definitivo. Se puede evidenciar que las relaciones laborales en la banca universal venezolana sufrieron cambios a partir del proceso de fusiones y que esto generó un nuevo trabajador bancario con características especiales y flexibilizadas.

\section{Evolución de las fusiones a nivel internacional}

Se presenta en el ámbito mundial una importante recomposición del capital 
con características globales, una nueva definición de los procesos globales y productivos, cambios tecnológicos importantes, nuevas formas de organización económica, y de división del trabajo y emergencia del neoliberalismo. Los sectores claves en este proceso fueron el financiero, la industria electrónica y las telecomunicaciones, los tres que se hermanaron y compartieron un lugar estratégico en la definición económica del mundo a partir de entonces (Bouzas, 2003: 56-57).

Es evidente que la banca latinoamericana desarrolla sus actividades hoy dentro de un marco legal diferente. Un gran porcentaje de los países latinoamericanos han introducido cambios significativos en lo correspondiente a los textos legales. Estos cambios se deben a razones como: las crisis de los sistemas bancarios, las nuevas características del entorno económico internacional, disponibilidad de nuevas tecnologías y el progreso a nivel internacional en materia de regulación y de supervisión bancaria (Aguirre, 1998). Ahora las limitaciones regulatorias al sector bancario son establecidas de manera internacional mediante mecanismos de concentración para todos los bancos en términos de los acuerdos y recomendaciones de Basilea y quedan asi integrados los mercados nacionales a los circuitos mundiales de capital a través de un sistema financiero global interconectado (Bouzas, 2003).

La banca a nivel mundial ha sido testigo en estos años de una ola de fusio- nes y adquisiciones enmarcadas dentro del proceso de globalización financiera que se viene gestando y de las estrategias de modernización asumidas por el sector. En el período 95-99 se materializaron en el mundo industrializado más de 4.700 transacciones de compra-venta referida a bancos e instituciones financieras. Estados Unidos concentró la mayor parte, registrando 3.585 operaciones, la Euro Zona fue escenario de 963 operaciones, y Japón de 141. Latinoamérica no escapa al centrifugado fusionario ${ }^{3}$. Brasil, Venezuela, Argentina y Chile son las economías que más reestructuraciones bancarias han experimentado en las últimas dos décadas (Ruiz, 2001:1).

Así mismo la OIT (2001:3-4) afirma que los países desarrollados son los vendedores y compradores más importantes en las operaciones de fusiones y adquisiciones ( $F \& A$ ) transfronterizas; en 1998-1999 realizaron respectivamente cerca del 90 por ciento y del 95 por ciento de las ventas/compras. La mayor parte (el $70 \%$ ) del 5 al $10 \%$ de las ventas/compras en que intervienen países en desarrollo tiene su origen en América Latina y el Caribe.

Los requerimientos internacionales del sector, el obligado proceso de internacionalización de la banca, derrumba fronteras y nos coloca en un proceso de cambio estructural y cultural en el que habremos de asimilar los nuevos esquemas de relaciones sociales impuestos por la lenta pero inevitable globalización económica estos países. 
y particularmente la financiera (Bouzas, 2003:19).

La oleada de fusiones y adquisiciones llevadas a cabo en la banca internacional ha provocado la eliminación de miles de puestos de trabajo en un sector caracterizado por la estabilidad laboral. Según un documento preparado por la Oficina Internacional del Trabajo (OIT, 2001); en Estados Unidos el número de bancos comerciales y holdings se redujo en casi un $30 \%$ entre 1988 y 1997. En tanto, el sector financiero de Europa Occidental perdió un mínimo de $\mathbf{4 3 0}$ mil empleos durante las década de los noventa. Sólo los bancos británicos redujeron su cifra total de empleados en 150 mil y cerraron una cuarta parte de su red de sucursales. En los países escandinavos, el sector bancario perdió entre 1995 y 1999 un 30\% de sus trabajadores. En Finlandia se llegó incluso al 50\%. Mientras, en Francia y Alemania las leyes protectoras de los empleados de banca amortiguaron las pérdidas sectoriales (Ruiz, 2001:3).

El que la actividad bancaria se diversifique es claro síntoma de que la reestructuración del sistema financiero exige menos profesionales y tal vez más especializados, en un campo donde la reducción y la rotación laboral se han intensificado en los últimos diez años. Según Ruiz (2001:1) "las grandes fusiones y adquisiciones van inevitablemente acompañadas por anuncios de despidos masivos. Menos instituciones financieras, pero de mayor tamaño y con una planta de funcionarios recortada, será la constante del sistema bancario mundial en el siglo XXI". Un fenómeno de pro y contras, y es que en una economía globalizada donde ganar posiciones es cada vez más difícil, tecnologías y fusiones son sinónimo tanto de supervivencia empresarial como de reducción de personal.

Según la OIT (2001), en Asia el número de bancos comerciales pasó de 26 a 17; además, se cerraron una de cada siete sucursales (en un total de 1.100). Estas medidas se tradujeron en el despido masivo de un cuarto del personal de la banca (34.000 personas de un total de 145.000) entre 1997 y 1999. En África la liberalización de los mercados financieros en ha tenido un ritmo mucho más intenso que el de la reforma de sus débiles instituciones financieras. Tras años de liberalización, reestructuración y otras reformas, el sector se ha fortalecido. Sin embargo, muchos observadores consideran que para que el sector sea competitivo habrá que seguir impulsando el proceso de privatización y fusiones.

\section{Evolución del sistema financiero venezolano. Período 1994-2006}

El proceso de concentración y globalización financiera, no es ajeno a los movimientos incipientes ocurridos en Venezuela luego de la crisis financiera de 1994, en el cual se observó un proceso de fusiones que no tiene antecedentes comparables. Los bancos comerciales y universales son los que han implantado con mayor frecuencia la fusión como una estrategia para permanecer en el mercado. A partir de 1994, en Venezuela se ha dado una intensificación de las fusiones, las cuales en una primera fase fueron por la vía de intragrupos para conformar los llamados Bancos Universales y, en una segunda fase se produjeron fusiones in- 
tergrupos para conformar grandes conglomerados financieros y que se han denominado megafusiones (Bracho et al., 2002: 302-311). El tipo de fusión que ha prevalecido en Venezuela es por absorción, ya que los grandes bancos han adquirido los bancos más pequeños permaneciendo la entidad del grupo financiero más poderoso.

De hecho como puede observarse en la Figura 1, en Venezuela el número de instituciones se ha reducido significativamente, con el riesgo de una concentración bancaria. Para 1994 existían en el sistema financiero venezolano 114 instituciones, en el 2000 disminuyen a 84 con un predominio de los bancos comerciales y universales y la aparición de los bancos con leyes especiales como el Banco Industrial de Venezuela, Banap Instituto municipal de crédito popular y Banco de Comercio Exterior. Para el 2002 el total de las instituciones se ubican en 77 de las cuales 69 eran privadas y 8 de carácter estatal (Armas, 2001: 38).

La tendencia del sistema financiero venezolano apunta a un cambio en la estructura como consecuencia de las fusiones, en los cuales disminuye los bancos comerciales y entidades de ahorro y prestamos, dándole paso a los bancos universales. Como puede observarse en la Figura 2 para 1996 existían 123 instituciones de las cuales sólo 2 eran bancos universales y en el 2006 el sistema financiero cuenta con 55 instituciones de las cuales 22 son bancos universales; esto representa una reducción para este periodo de $44.72 \%$, como consecuencia de 38 fusiones de las que 30 fueron para transformarse a banca universal.

Las fusiones que se han llevado a cabo en el sistema bancario venezolano persiguen disminuir los costos de trans-

Figura 1

\section{Evolución del sistema financiero venezolano. 1994-2002}

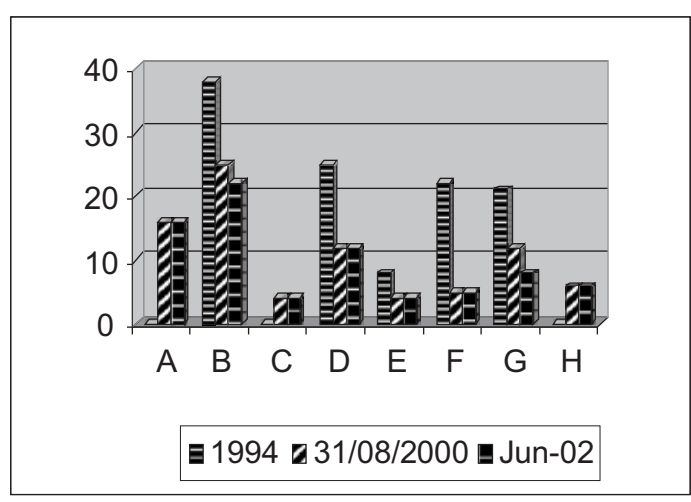

\begin{tabular}{|r|r|r|c|}
\hline & $\mathbf{1 9 9 4}$ & $\mathbf{3 1 / 0 8 / 2 0 0 0}$ & Jun-02 \\
\hline$A$ & 0 & 16 & 16 \\
\hline$B$ & 38 & 25 & 22 \\
\hline$C$ & 0 & 4 & 4 \\
\hline$D$ & 25 & 12 & 12 \\
\hline$E$ & 8 & 4 & 4 \\
\hline$F$ & 22 & 5 & 5 \\
\hline$G$ & 21 & 12 & 8 \\
\hline$H$ & 0 & 6 & 6 \\
\hline
\end{tabular}

Total: 114

84

77

Donde: A: BANCOS UNIVERSALES, B: BANCOS COMERCIALES, C: BANCOS CON LEYES ESPECIALES, D: BANCOS DE INVERSION, E: BANCOS HIPOTECARIOS, F: ARRENDADORAS FINANCIERAS, G: ENTIDADES DE AHORRO Y PRESTAMO, H: FONDOS DEL MERCADO MONETARIO. 


\section{Figura 2 \\ Resumen de los Procesos \\ Sistema Financiero Venezolano. 1996-2006}

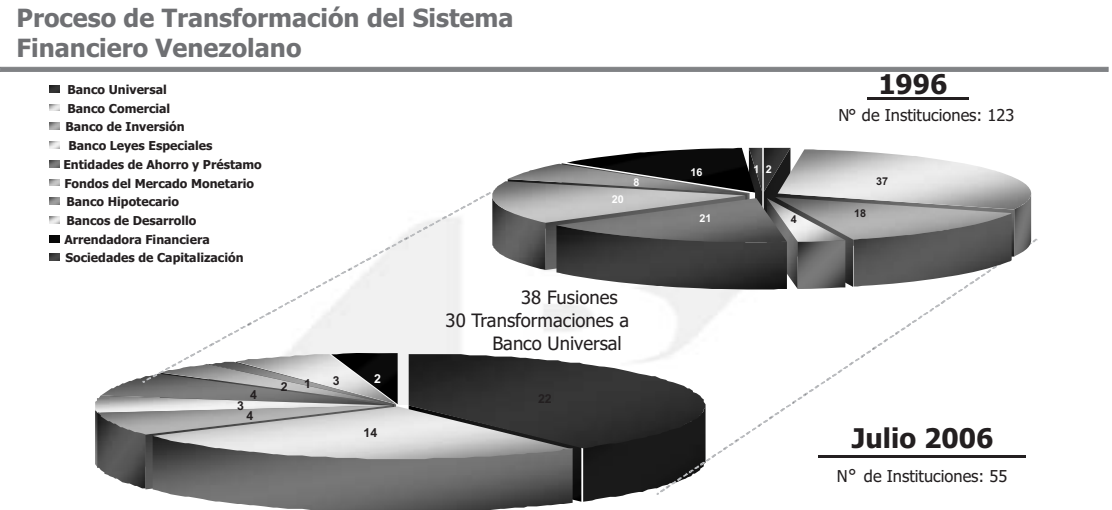

Fuente: Sudeban. Asociacion Bancaria de Venezuela (2002:3).

formación, aprovechar las economías de escala, fortalecer el patrimonio y las operaciones tecnológicas y operativas con miras a aumentar la rentabilidad en sus actividades.

Según la OIT (2001: 6), "la liberalización y los adelantos tecnológicos están empujando el sector hacia una mundialización creciente en la que las $F \& A^{4}$ son a la vez causa y consecuencia. Los observadores creen que las F\&A transfronterizas en los servicios financieros constituyen la próxima etapa, una vez que se haya completado la concentración interna, y que la tendencia a una concentración cada vez mayor ha de detenerse únicamente cuando no haya más objetivos de adquisición (......). El rápido desarrollo de Internet hace que las predicciones a mediano plazo relativas a la naturaleza y al ritmo de las F\&A en los servicios financieros sean particularmente arriesgadas ya que la tecnología está incrementando la capacidad de los nuevos actores que ingresan al sector para competir por ciertos «nichos» de mercado y está intensificando también la presión de la competencia que está contribuyendo a la concentración sectorial".

En Venezuela se produjo un cambio en la Ley General de Bancos y otras Instituciones Financieras de 1997 en la que se le daba ya entrada al capital extranjero para promover una mayor competencia entre las instituciones financieras y en la Ley del año 2001 se promovieron directamente todas las fusiones y adquisiciones en los artículos 76, 77, 78 y 79 ; en el primero se autoriza el funcionamiento como banco universal a los ban- 
cos que se fusionen o se transformen, en el segundo se específica la documentación necesaria para la fusión y transformación y el quórum y el efecto inmediato de la fusión respectivamente. Las crisis financieras, a menudo resultantes de programas de liberalización mal aplicados, han impulsado las fusiones y adquisiciones. La desreglamentación ha intensificado la competencia mediante dos mecanismos: abriendo el sector para que entren nuevas empresas (bancos extranjeros e instituciones distintas de los bancos) y aligerando la reglamentación que impedía antes la expansión en diferentes segmentos financieros (servicios bancarios para particulares, bancos de inversiones/bancos mercantiles, etc.), y mediante el estímulo al desarrollo de nuevos productos, y la convergencia en los servicios proporcionados por diferentes tipos de operadores financieros. La intervención del Estado puede ser también otro factor determinante de la concentración del sector financiero cuando los gobiernos actúan como vendedores en el proceso de privatización (OIT, 2001:10-11).

El proceso de fusiones a partir de 1996 no tiene antecedentes históricos, ya que hasta 1995 fueron eventos poco frecuentes en nuestro país. Las escasas fusiones registradas en los años 60 tienen su origen en la concepción del negocio bancario, que funcionaba prácticamente como una empresa familiar (banca de un solo dueño), donde había pocas economías de escala, un ambiente protegido que permitía bancos de tamaño reducido. A partir de 1996 los procesos de fusiones se deben a crear bancos universales, sin cambio de propietarios y el otro por la in- corporación de bancos simultáneamente a su adquisición (BCV, 2001).

En Venezuela la concentración bancaria originada por los procesos de fusiones no tienen una lata concentración, tomando como referencia el índice de Herfindahl-Hirschman; el cual se obtiene a partir de elevar al cuadrado la cuota de mercado de un banco, basándose tanto en cifras reales como hipotéticas que se darían luego de la fusión. Existe un nivel de advertencia cada vez que una fusión resulte en una cuota de mercado superior al $33 \%$. Aún tomando en cuenta las fusiones anunciadas, el banco que tendría la mayor cuota de mercado registraría un puntaje de 1.766 puntos (el índice oscila entre 0 y 10.000 puntos, este último caso sería un monopolio absoluto), esta cifra muestra que los procesos que se estaban verificando en Venezuela no generaban una investigación especial. Comparando con otros países, el nivel de concentración bancaria, expresado como el porcentaje de los depósitos totales que manejan los cinco (5) principales bancos del sistema, se ubica por debajo de lo observado en Latinoamérica. Siendo este un nivel de concentración aceptable (BCV, 2001: 4). Esto puede observarse en la Tabla 1. Esto se traduce en una mayor consolidación de la banca de menor talla, procurando así del mercado de los bancos con debilidad patrimonial.

La evolución histórica de las fusiones que se dieron en Venezuela desde 1996 hasta el 2006 en el sistema financiero venezolano, se describen a continuación en los Cuadros 1 y 2, con las respectivas instituciones que se fusionaron y los cambios que se dieron para conformar 


\section{Tabla 1}

Evolución Histórica del mercado

\begin{tabular}{ccc}
\hline Años & Cuota $\left(^{*}\right)$ & No. de Bancos \\
\hline 1940 & $84,3 \%$ & 12 \\
1975 & $40,4 \%$ & 32 \\
1985 & $51,1 \%$ & 38 \\
1990 & $55,1 \%$ & 42 \\
1995 & $58,3 \%$ & 39 \\
2000 & $56,1 \%$ & 41 \\
\hline
\end{tabular}

Fuente: BCV, 2001: 4. $\left({ }^{*}\right)$ Concentración: porcentaje del total de depósitos que tienen los primeros 5 bancos.

los bancos universales y los bancos comerciales que están operando actualmente en el país.

En el Cuadro 2 se enumeran los bancos clasificados por subsistema para Julio del 2006 del Sistema Bancario Venezolano. Según la Sudeban (2006) de las 36 instituciones de la banca universal y comercial, la banca extranjera posee el $30 \%$ de los activos del sistema.

En la Figura 3 se observa esquemáticamente que la principal causa de las fusiones en Venezuela a partir de 1996 fue la crisis financiera del sector de 1994 y que el $93.6 \%$ era de capital nacional y con muy poca participación del estado. Posteriormente en 1996 empieza la consolidación del sistema financiero venezolano con la apertura al capital extranjero y este aumentó su participación de 0,7\% a $22 \%$. Al igual que se observa el incremento de participación del estado venezolano al pasar de $5,7 \%$ a $26,5 \%$.
En el 2006 se visualiza un sistema financiero fortalecido con una composición del capital nacional de $59 \%$, un capital extranjero de $29,9 \%$ y del estado de un $11,1 \%$. Según la Asociación Bancaria de Venezuela (2006) los depósitos totales se incrementaron para el 2006 al pasar de Bs.17.187 millardos a Bs.90.862 millardos para el 2003, la cartera de créditos también se incrementó al pasar de Bs. 9.358 millardos a Bs. 46.153 millardos para el mismo periodo. La intermediación también se incrementó al pasar en el 2003 de 36,3 a 50,8; aunque en el último año se redujera por las políticas de encaje que estableció el Banco Central de Venezuela a partir de marzo de 2006 (Dinero, 2007: 30). El índice de morosidad disminuyó en este periodo de 4,6 a 1,3 y el $\mathrm{ROE}^{5}$ real que mide el nivel de rendimiento del patrimonio de los bancos, ósea cuantifica el retorno sobre la inversión de los accionistas se ubica a partir de 2003 en 43,3 , valor muy superior a los anteriores a los procesos de fusiones para el 2000. Si bien es cierto este último disminuyó para julio de 2006 31,4; este se ubica en valores superiores a los alcanzados antes del proceso de fusión. Se visualiza también un incremento de los activos totales y una concentración en menos instituciones financieras.

Todos estos procesos de fusiones bancarias sin duda alguna inciden sobre la cantidad y calidad de las relaciones laborales al crear un trabajador con carac-

5 Resultado Neto/Patrimonio Promedio, criterio metodológico aplicado por la Superintendencia de Bancos (Sudaban). 


\section{Cuadro 1 \\ Evolución de las fusiones bancarias en Venezuela. Año 1996-2006 A Bancos Universales}

\section{Fusiones y Transformación a Bancos Universales}

1. Banco Provincial S.A. Banco Universal

2. Banco Mercantil C.A.

Banco Universal

\section{FIVENEZ}

Banco Universal

4. Citibank, N.A.

Banco Universal

5. Banco Internacional Interbank, Banco

Universal

6. Banco Exterior C.A. Banco Universal

7. Banco Caroní C.A.

Banco Universal

\section{Características y fecha de la fusión}

\section{6-11-1996}

Fusión por absorción del Banco Provincial S.A. con: Arrendadora Provincial, Sociedad de Arrendamiento Financiero, C.A., Fondo Provincial de Activos Líquidos, C.A. (En fecha 1ro. De Julio de 1996, el Fondo de Activos Líquidos Finalven, quedó igualmente fusionado con el Fondo Provincial de Activos Líquidos, C.A. Posteriormente con Banco Popular y de los Andes, C.A. (1998). Banco de Occidente, C.A. (1999); Banco de Lara, C.A. (2000); Inversiones BAN-PRO, C.A. (2002)

31-12-1996

Fusión por absorción DEL Banco Mercantil, S.A.C.A. con:

Banco de Inversión Mercantil, Banco Hipotecario Mercantil, Arrendadora Mercantil en 1997; Interbank, C.A. (2000)

20-12-1996

Fusión por absorción con:

FIVENEZ S.A.C.A. Banco Universal con las siguientes instituciones: FIVENEZ, Arrendadora Financiera, S.A. FIVENE Banco Hipotecario, S.A. Inversora FIVENEZ, S.A.

24-01-1997

\section{9-04-1997}

Fusión por absorción con:

Arrendadora Internacional, C.A., Fondo Internacional, C.A. Resolución por la cual se autoriza la fusión por absorción de Venezolana, Entidad de Ahorro y Préstamo, C.A. por parte de Interbank, C.A. Banco Universal (24-08-2000)

03-04-1997

Fusión por absorción con:

Arrendadora Exterior C.A. Sociedad de Arrendamiento Financiero, Fondo Exterior C.A.

19-06-1997 


\section{Cuadro 1 \\ Evolución de las fusiones bancarias en Venezuela. Año 1996-2006 A Bancos Universales (Continuación)}

\section{Banesco}

Banco Universal C.A.

9. Banco Orinoco C.A.

Banco Universal

10. Banco del Caribe, C.A. Banco Universal

11. Banco Caracas C.A. Banco Universal

12. Corp Banca, C.A.

13. Bolívar Banco Universal

14. Fondo Común C.A. Banco Universal

\section{2-08-1997}

Fusión por absorción con:

Banesco Arrendamiento Financiero y Banesco Fondo de Activos Líquidos en 1997; Unibanca Banco universal (2002) producto de la fusión entre Banco Unión, C.A., fundado en 1946 y Caja Familia, Entidad de Ahorro y Préstamo, C.A., constituida originalmente en 1963 con el nombre de La Industrial, Entidad de Ahorro y Préstamo, fusionada en 1998 con El Porvenir, Entidad de Ahorro y Préstamo, C.A., Caja Popular Falcón-Zulia, Entidad de Ahorro y Préstamo, C.A., Maracay, Entidad de Ahorro y Préstamo, C.A. y Bancarios, Entidad de Ahorro y Préstamo, C.A., y nuevamente fusionada en el año 2000 con La Primera, Entidad de Ahorro y Préstamo, C.A.; Banco Hipotecario Unido, S.A: (2002); Fondo Unión C.A. (2002); Banco de Inversión Unión, C.A. (2002).

08-07-1997

Fusión por absorción con el Banco Orinoco S.A.C.A. con: Banco Hipotecario de Orinoco, C.A., Arrendadora del Orinoco, Arrendamiento Financiero, C.A. y Fondo de Inversiones Orinoco.

06-04-1998

Fusión por absorción con:

Banco de Inversión del Caribe y Fondo de Activos Líquidos del Caribe en 1998.

22-12-1998

Fusión por absorción del Banco Caracas, C.A con:

Banco de Inversión Bancaracas, C.A., Activos Líquidos Bancaracas, C.A. Posteriormente fusión de FIVENEZ, S.A.C.A., Banco Universal por absorción con el Banco Caracas, C.A. Banco Universal

\section{6-09-1999}

Fusión por absorción con:

Corp Banca de Inversión, Corp Banca Hipotecario, Corp Arrendadora Financiera, Corp Fondo de Activos Líquidos, C.A. y Sociedad Anónima de Arrendamiento Financiero en 1999 y del Banco Orinoco, S.A.C.A. Banco Universal por parte del Grupo Corp Banca, C.A.

18-11-1999

Fusión por absorción del Fondo Financiero Bolívar, C.A. y Ávila Sociedad de Capitalización por Bolívar Banco de Inversión, C.A.

21-12-2000

Fusión por absorción con:

Banco República C.A. Banco Universal (2000); Fondo Común Entidad de Ahorro y Préstamo, S.A. (2000) 


\section{Cuadro 1 \\ Evolución de las fusiones bancarias en Venezuela. Año 1996-2006 A Bancos Universales (Continuación)}

\section{Banco Occidental \\ de Descuento, \\ Banco Universal C.A.}

16. Banco Sofitasa

Banco Universal, C.A.

17. Del Sur

Banco Universal C.A.
13-11-2002?

Fusión por absorción con:

Banco Occidental de Descuento S.A.C.A., Fondo de Activos Líquidos Banco Occidental, C.A. y Banco Monagas, C.A. por parte de Normal Bank C.A. Banco Universal, autorizado el 13-11-2002

18-10-2001

Fusión por absorción con:

Banco Sofitasa,c.a. Fondo Sofitasa, C.A. y Arrendadora Sofitasa, C.A.

18-10-2001

Del Sur Banco de Inversión C.A.

Fusión por absorción con:

Mérida Entidad de Ahorro y Préstamo C.A. (2001), Del Sur Entidad de Ahorro y Préstamo C.A. (2001)

18. Venezolano de Crédito S.A. 26-12-2001

Banco Universal

Fusión por absorción con:

Soficredito banco de Inversión, C.A. (2001), Sogecredito C.A. de arrendamiento financiero (2001)

19. Banco de Venezuela S.A. Banco Universal

28-08-1997

Fusión por absorción con:

Banco Caracas C.A. Banco Universal (2002)

20. Banco Canarias de

Venezuela Banco Universal

Fusión por absorción con:

Margarita, Entidad de Ahorro y Préstamo, C.A.

Fuente: Superintendencia de Bancos y Otras Instituciones Financieras. Gerencia Técnica y Normas Prudenciales Dpto. Estudios Especiales. Bracho Yajaira, et al (2002: 309). (Superintendencia de Bancos) y Organización Propia.

terísticas muy particulares a nivel mundial. En Venezuela, estos procesos también generaron cambios en las relaciones laborales que se tradujeron en unas nuevas características del trabajador bancario.

\section{Nuevas características del trabajador bancario. Consecuencias laborales de las fusiones bancarias}

Algunas características de las relaciones laborales como el descentramien- to del trabajo, la segmentación de la mano de obra, la inestabilidad en el empleo, la individualización de las relaciones de trabajo y el abaratamiento del costo de trabajo (Ermida, 1999:139) han cambiado en este proceso de fusiones en el sector financiero, produciéndose un clima organizacional lo suficientemente tenso que se ha traducido en algunas incidencias para el éxito de las organizaciones financieras fusionadas.

La reestructuración que siempre acompaña las fusiones y adquisiciones 


\section{Cuadro 2 \\ Evolución de las fusiones bancarias en Venezuela. Año 1996-2006 De los Bancos Comerciales}

\section{Fusiones y Transformación} de los Bancos Comerciales

1. Banco Nacional de Crédito, C.A.

2. Eurobanco Banco Comercial, C.A.

3. Banco Standard Chartered (sucursal Venezuela).

4. Helm Bank de Venezuela, S.A. Banco Comercial Regional.

5. Nuevo Mundo

Banco Comercial, C.A.

6. Banco de la Gente Emprendedora (BANGENTE, C.A.)

7. Banco Andino Venezolano, C.A.

8. Banco Confederado, S.A.

9. ABN AMOR Bank N.V.

Banco Comercial.

10. Banco Galicia de Venezuela, C.A. Banco Comercial

11. Bolívar Banco, C.A

\section{Características y fecha de la fusión}

El cambio de denominación del Banco Tequendama, C.A. a Banco Nacional de Crédito, C.A. fue autorizada el 27-03-2003.

Fusión de Italcambio Casa de Cambio, Italfin Banco de Inversión y FAL para dar origen al Banco Comercial Italcambio fue autorizada el 10-10-1996.

El cambio de denominación de Sucursal de Banco Exterior y de los Andes, S.A. "Extebandes" a Banco Standard Chartered fue autorizada el 6-04-1998.

Su funcionamiento fue autorizado el 27-03-2003.

El cambio de objeto social de Aveauto Arredadora Financiera, C.A. a Banco Comercial fue autorizada el 17-12-1998.

El cambio de objeto social de la Arrendadora Financiera del Caribe, C.A. a Banco Comercial fue autorizada el 04-11-1998.

El cambio de denominación a Banco del Pueblo, C.A. se dejó sin efecto en la Asamblea Extraordinaria de Accionistas el 21-10-1999.

El cambio de objeto social de banco universal a banco comercial regional fue autorizada el 20-10-2001.

El cambio social de banco universal a banco comercial fue autorizada el 31-12-2001.

El cambio de denominación de Sofimeca Banco de Inversión, C.A. a Banco Galicia de Venezuela, C.A. Banco de Inversión fue autorizada el 5-05-2000 y este a Banco Comercial fue autorizada el 20 de Febrero de 2002.

La transformación a banco universal fue autorizada el 18-11-1999 (fusión por absorción con Fondo Financiero Bolívar y Ávila Sociedad de Capitalización). El cambio de objeto social a banco comercial fue autorizado el 29-06-2002.

Fuente: Superintendencia de Bancos y Otras Instituciones Financieras. Gerencia Técnica y Normas Prudenciales Dpto. Estudios Especiales. 


\section{Cuadro 3. Sistema Bancario Nombre de Instituciones según subsistema. Julio 2006}

\section{Banca Universal}

1. Banco Mercantil, C.A. Banco Universal

2. Banco de Venezuela, S.A. Banco Universal

3. Banesco Banco Universal, C.A.

4. Banco Provincial, S.A. Banco Universal

5. Banco Occid. de Descuento, Banco Universal, C.A.

6. Banco del Caribe, C.A. Banco Universal

7. Banco Exterior, C.A. Banco Universal

8. Banco Caroní, C.A. Banco Universal

9. Fondo Común, C.A. Banco Universal

10. Banco Nacional de Crédito, C.A.

11. Citibank, N.A. Banco Universal

12. Banco Canarias de Vzla., Banco Universal, C.A.

13. Venezolano de Crédito, S.A. Banco Universal

14. Corp Banca, C.A. Banco Universal

15. C.A. Central, Banco Universal

16. Del Sur Banco Universal, C.A.

17. Provivienda, Banco Universal, C.A.

18. Banco Sofitasa, Banco Universal, C.A.

19. Totalbank,C.A. Banco Universal

20. Banco de Fomento Regional los Andes, C.A. (BANFOANDES)

21. Banco del Tesoro, C.A. Banco Universal

22. Banco Agrícola de Venezuela, C.A. Banco Universal

\section{Banca Comercial}

1. Bancoro,C.A.

2. Banco Federal,C.A.

3. Banco Guayana, C.A.

4. Bolivar Banco, C.A.

5. Banco Confederado, S.A.

6. Banco Plaza, C.A.

7. Banvalor Banco Comercial, C.A.

8. Stanford Bank,S.A. Banco Comercial

9. ABN Amro Bank,N.V (Sucursal Venezuela)

10. Inverunion Banco Comercial,C.A.

11. Bannote (BANORTE) Banco Comercial,C.A.

12. Helm Bank de Venezuela,S.A. Banco Regional Comercial

13. Financorp Banco Comercial,C.A. 1_/

14. Banco de Exportación y Comercio,C.A.

\section{Banca con Leyes Especiales}

1. Banco Industrial de Venezuela ,C.A. 2_ I

2. Instituto Municipal de Crédito Popular (IMPC)

3. Banco de Comercio Exterior, C.A. (BANCOEX)

4. Banco Nacional de Vivienda y Hábitat (BANAVIH)

\section{Banca de Desarrollo}

1. Banco de Desarrollo Económico y Social de Venezuela (BANDES)

2. Banco de la Gente emprendedora (BANGENTE), CA. 2_

3. Banco de Desarrollo del Microempresario, C.A. 2_

\section{Banca de Inversión}

1. Baninvest Banco de Inversión, C.A.

2. Sofioccidente Banco de Inversión, C.A.

3. Federal Banco de Inversión, C.A.

4. Banco de Inversión Industrial de Venezuela, C.A. (FIVCA)

\section{Banca Hipotecaria}

1. Banco Hipotecario Activo, C.A.

2. Banco Hipotecario de Inversión Turística de Venezuela, C.A. (INVERBANCO)

\section{Empresas de Arrendamiento Financiero}

1. Arrendadora Financiera Empresarial, C.A. ANFICO

\section{Entidades de Ahorro y Préstamo}

1. Mi Casa, Entidad de Ahorro y Préstamo, C.A.

2. Casa Propia, Entidad de Ahorro y Préstamo, C.A.

3. Banplus Entidad de Ahorro y Préstamo, C.A.

\section{Fondos del Mercado Monetario}

1. Federal Fondo del Mercado Monetario, C.A.

2. Participaciones Vencred ,S.A..

Fuente: Asobanca, 2006. Superintendencia de Bancos y otras instituciones financieras. Gerencia General Técnica - Gerencia de Estadísticas y publicaciones. 1_/ Hasta el 31 de marzo de 2006 FINANCORP Banco de Inversión, C.A. 2_ A los fines estadísticos se incluye como Banca Comercial. 
en el sector financiero, generalmente se traduce en la desaparición de puestos de trabajo en las empresas afectadas, a veces en gran escala, y en un paso del empleo tradicional a tiempo completo al empleo temporal y ocasional, y otras formas atípicas de empleo. Aunque las pérdidas de puestos de trabajo afectan a la plantilla en toda su jerarquía, esas pérdidas afectan desproporcionadamente a las mujeres y a otros empleados de los niveles inferiores, en particular, en las economías más pobres, donde muy probablemente sus lugares de trabajo se verán afectados por la racionalización. Por supuesto estas son consecuencias negativas que puedan tener todos los trabajadores del sector. Algunas de estas consecuencias se traducen en la terminación de la relación laboral, traslados internos, la restricción de las horas extraordinarias y la reducción de las horas de trabajo normales. Existe otro elemento importante, como son las condiciones de trabajo y empleo que de alguna manera reflejan los cambios en la organización del trabajo; existe además una inseguridad laboral y una mayor carga en la empresa fusionada. Las fusiones y adquisiciones invariablemente requieren que diferentes sistemas y procedimientos empresariales se integren con vistas a armonizar varios aspectos de los términos y condiciones de empleo a fin de asegurar prácticas comunes en la nueva organización fusionada. Si no existe una fuerza de trabajo calificada, estable y motivada no se percibirá el éxito necesario en toda organización empresarial (OIT, 2001: 2).

Lo antes expuesto ratifica que existe un nuevo tipo de cesante que aparece como consecuencia de las fusiones. His- tóricamente, la desocupación estaba concentrada en personas con años de escolaridad relativamente bajos, y también observábamos que en la estructura del mercado laboral las personas en general tenían trabajos de por vida. Eso cambió para todas las economías y para las distintas actividades. Las economías actuales exigen un proceso de aprendizaje, actualización y capacitación continua. Sin embargo el desarrollo tecnológico y las fusiones generan desocupación, de una mano de obra bien calificada.

Al respecto Jinkings (2002: 3) piensa que los bancarios como millones de trabajadores afectados por la reestructuración contemporánea del capital, experimentan la inestabilidad del empleo y la intensificación del trabajo en su vida cotidiana. En tanto segmento de la clase trabajadora directamente vinculado a los movimientos comandados por el capital financiero -lidiando en su actividad diaria con la fracción del capital que se valoriza conservando la forma dinero-, los bancarios viven de modo singular las transformaciones del capitalismo mundializado.

Adicionalmente Jinkings (2002:5) enuncia que existen unos elementos nuevos en la reorganización del trabajo en los bancos el cual esta caracterizado por un proceso intenso de reorganización operacional y del trabajo para adaptarlos a la manera en la cual el capital se reproduce.

También es importante destacar el desarrollo tecnológico y científico que se ha dado en el sector financiero que han incrementado las estrategias de mercado y de reorganización del trabajo. Al respecto Jinkings (2002:9) enuncia que con el soporte teleinformático se desactivan los grandes centros de procesamiento de 
datos, de servicio y de compensación que reunían numerosos contingentes asalariados en los años 60,70 y 80 . Paralelamente, el paso de la atención tradicional en las agencias bancarias a la atención electrónica -a través de centrales telefónicas, cajeros automáticos, internet y sistemas homebanking (sistemas electrónicos que permiten la conexión de la computadora del cliente a la del banco) y la progresiva sustitución del papel-dinero por la tarjeta electrónica, convierten a las agencias en tiendas informatizadas de "productos" y servicios financieros-. Estos procesos de reestructuración permiten incrementos de la productividad del trabajo como resultado de la reducción de la plantilla de los trabajadores del sector. Si bien es cierto el acceso telefónico, los cajeros automáticos y las computadoras en las oficinas existen hace años; lo realmente novedoso es la banca digital que ha modificado la organización del trabajo en las oficinas y el número de personal para atender a los clientes.

Tanto los nuevos productos financieros como la aplicación de nuevas tecnologías han transformado la organización del trabajo y los requerimientos profesionales y el perfil del puesto. La tradicional forma de trabajar de las distintas bancas y sus sucursales, en la que los empleados realizaban operaciones repetitivas y sistematizadas al grado de que las verificaban de manera mecánica, mientras conversaban o atendías otras actividades, ha sido transformada y el nuevo trabajador se encuentra armado con un terminal de computadora conectada a un centro de computo en el que se sistematizan las operaciones, integran los resultados, realizan servicios múltiples en el pasado fragmentados, esto en busca de una mayor eficiencia y competitividad. Ha llegado incluso el momento de jubilar el reloj checador, ya que el control de tiempos y movimientos y cualquier otro mecanismo de los que en el taylorismo y en el fordismo se utilizaron para controlar el trabajo se hacen innecesarios; la maquina registra y puede reportar de distintas maneras los indicadores de rendimiento del trabajo que se quieran conocer, control que se enfatiza cada vez más a través del teletrabajo. Las actividades se realizan ahora desde el propio domicilio del trabajador y reclaman una nueva calificación del empleado bancario con una permanente actualización. Estas nuevas condiciones de trabajo someten al trabajador a una continua tensión, un permanente estrés por temor de llegar a ser desplazado; agudiza el individualismo en la búsqueda de opciones laborales, reduce los espacios de participación de la organización sindical ante la permanente competencia individual (Bouzas, 2003).

La política empresarial en el sector bancario, implica ampliar los horarios sin límites, reducir el número de plazas sindicalizadas, incrementar las formas de trabajo precario, la formación profesional; implica la determinación unilateral de los ascensos y la definición salarial mediante mecanismos que establece la empresa en función de su idea de productividad y salario flexible (Villamar, 1991 citado por Bouzas, 2003: 76).

Algunas características de las relaciones laborales tradicionales y las nuevas condiciones del trabajo en el sector bancario según Jinkings (2002), Bouzas (2003) y Quiñones (2005) , se pueden observar en el Cuadro 4. 


\section{Cuadro 4 \\ Características de las relaciones laborales del sector bancario tradicional y del sector bancario actual}

helacion Laboral Tradicional

Criterios

Capacitación

o Calificación

Toma de decisiones

Antigüedad

Control y gestión

del trabajo

Organización del trabajo

Evaluación del
desempeño

Canales de distribución

Participación de los Sindicatos

Remuneraciones

Movilidad Funcional o Tareas
Relación Laboral Tradicional

Relación Laboral Actual

Sin calificación y basado en el aprendizaje en el puesto de trabajo, con conocimientos básicos.

Tradicional y repetitivo

Carreras laborales constituidas sobre la permanencia en el puesto.

Retiro por alcanzar la edad jubilatoria

Manual

Estructuras horizontales y con líneas de mando largas.

Forma del trabajo basada en el binomio base-confianza.

Evaluación de la tarea no ligada al salario.

Tradicional, en la oficina bancaria.

Sindicato fuerte y con representatividad.

Salario base conreinvindicaciones salariales como: pago de comida, transporte y horas extras.

Funciones minuciosamente delimitadas

Plantillas de gran tamaño lo Universitario.

Proactivos dos financieros. lación o retiro voluntario. ductividad.

Vigilancia Jerárquica.

Uso del Timer. zontales equipos de trabajo.

Individual con base a metas online

Debilitamiento sindical

Patronal. productividad individual.

Polifuncionales
Amplios perfiles profesionales con una capitación mínimo TSU o Títu-

Bancarios vendedores, con capacidad para comprender los merca-

Programa de presión para la jubi-

Automatizado basado en la pro-

Líneas de mano cortas con controles inmediatos y estructuras hori-

Forma del trabajo basado en los

Home Banking, telemarketing y

Cuadro de remuneraciones con un salario base y prestaciones económicas fijas y variables con base a la

Plantillas reducidas. Despidos de los responsables de las tareas de infraestructuras de apoyo o de atención simplificada.

Movilidad Geográfica Fuerte arraigo geográfico Expectativas de movilidad geográfica intra e inter puestos.

Extendida y con un horario acorde a las necesidades institucionales

Fuente: Clasificación Propia a partir de Jinkins ( 2002 ), Bouzas (2003) y Quiñones (2005). 
A partir de las nuevas características en este artículo se pretende indagar cuales son estas características en la banca universal venezolana.

\section{Características de las relaciones laborares en el sistema financiero venezolano. Caso Banesco Banco Universal}

Banesco Banco Universal, es constituido originalmente en 1977 con el nombre de Banco Agroindustrial Venezolano, el cual en 1987 cambia su denominación a Banco Financiero, nuevamente cambiada en 1990 por Bancentro Banco Comercial, C.A. Es la institución financiera que ha sufrido el mayor número de fusiones en Venezuela. Este proceso comienza en el 2002, en una primera fase con las empresas de su grupo y posteriormente con Unibanca Banco Universal, C.A. (institución que fue el producto de la fusión del Banco Unión, C.A. y Caja Familia, Entidad de Ahorro y Préstamo, C.A.). Esto puede observarse en el Cuadro 2.

Banesco posee el $30 \%$ aproximadamente de la participación del subsector con el mayor número de trabajadores en oficinas y sucursales y agencias a partir del 2002. Adicionalmente también posee el mayor número de oficinas a nivel nacional. El subsector de banca privada de capital nacional con respecto al número de trabajadores posee el $63 \%$ del sector financiero venezolano y el subsector de la banca privada de capital extranjero posee el $35 \%$, ésto puede observarse en el Cuadro 4.

Hubo un incremento desde 1996 hasta el 2006 de los trabajadores en la banca universal; pero existe una disminu- ción del $82 \%$ en la banca comercial acompañado de los dramáticos descensos en las otras instituciones del sistema financiero, muchas veces superior al $80 \%$. En sentido general el sistema financiero venezolano tuvo una reducción global del $23 \%$ en el periodo analizado del número de los trabajadores.

En cuanto al número de oficinas desde 1998 hasta el 2006 hubo una disminución del 36,6\%. Destacándose las disminuciones en el número de oficinas de todas las instituciones del sistema financiero excepto en la banca universal. Esto conlleva a una flexibilización de tipo numérica en cuanto al número de trabajadores y de oficinas en el sistema financiero venezolano.

Este proceso puede indicar un reacomodo del sistema en cuanto a calificación del personal y crecimiento del mercado.

Los resultados del cuestionario aplicado y que recoge la posición de los trabajadores de Banesco Banco Universal en cuanto a las características de las relaciones laborales a partir del proceso de fusiones, sus causas y consecuencias fueron los siguientes:

1. Entre las causas que piensan los empleados que conllevaron a las fusiones de su institución financiera destacan: incrementar la cartera de clientes, adquirir una tecnología de punta y ser lideres del mercado, reacomodo del sistema financiero venezolano, ventajas competitivas, convertirse en banco universal, la crisis financiera, el prestigio de ambos bancos, relacionar los productos, las altas tasas de interés, entre otros. El elemento más importante es la crisis financiera con 
un $23,5 \%$ de las respuestas seguido de la búsqueda de solidez con un 14,3\%; la captación de una cartera de clientes y adquirir una tecnología de punta y ser lideres del mercado con un $9,5 \%$ c/u respectivamente.

2. La cultura organizacional estuvo influenciada por los cambios tecnológicos, por procesos de adaptación, por procesos del capital humano, conocimiento de los nuevos procesos, por romper paradigmas, pero fundamentalmente por la presencia de un choque de culturas lo cual representó un $30,8 \%$ de los encuestados.

3. Las estrategias de la gerencia en cuanto a las relaciones laborales fue la adaptación del personal a los nuevos productos con un $23,1 \%$; mejorar las estrategias para la atención del cliente; cambios estratégicos de personal, exigir mayor preparación académica con un $23,1 \%$; reuniones periódicas, inducción y adiestramiento en la nueva tecnología y adiestramiento y jornadas de integración con un $15,4 \%$.

4. En promedio creen que el numero de oficinas después de terminado el proceso de fusiones se incremento a nivel nacional en mas de 100 oficinas aunque en una primera fase se diera el cierre de muchas agencias, acompañada con una ola de despidos que en promedio el porcentaje se ubicó en un $51 \%$.

5. Las áreas en las cuales se produjo mayormente los despidos o que se eliminaron totalmente estuvo en los departamentos con gran número de personal con más del $26,3 \%$, seguido de las secretarias y recepcionistas con un $15,3 \%$, otras áreas fueron promociones, operativas, canal de ventas, taquilla, tarjetas de crédito, chequeristas, departamentos administrativos, gerencias y vicepresidencias y las oficinistas de agencias.

6. Los elementos tomados que fueron en consideración para abrir y cerrar agencias en primer lugar fueron: la ubicación geográfica, seguido por el número de operaciones y el nivel de captación y otros elementos serían si la agencia era alquilada, la zona de alto impacto, la comodidad y acceso a las oficinas y la rentabilidad de la agencia.

7. Los despidos de los empleados fueron influenciados en primer orden por la antigüedad y el nivel académico. Otros elementos de menor importancia según los empleados fue el minimizar los costos, la reducción de personal, las evaluaciones, los resultados de las auditorias, los errores internos y el desempeño.

8. Más del $50 \%$ de los encuestados piensan que se produjeron cambios en la jornada laboral y que esto estuvo influenciado por el entrenamiento del personal en los nuevos procesos, la atención a los clientes, el exceso de trabajo y el horario extendido por la cola de los clientes.

9. En promedio los empleados manifiestan que la jornada de trabajo tiene 8 horas diurnas, 1 hora nocturna y menos de 1 hora de descanso. El 100\% de los empleados tienen una jornada laboral total y no por turnos.

10. En general no tienen una visión de cuantas tareas en forma numérica realizaban antes y realizan después 
de la fusión; por lo que se puede deducir que siempre han tenido múltiples tareas. Lo que se puede visualizar es que la naturaleza de las tareas cambiaron influenciadas por los nuevos sistemas como por ejemplo el quick matic (entrega de tickets y direccionalidad de los clientes en la cola para ser atendidos), la atención a los cajeros automáticos y la atención personaliza y especializada de los clientes.

11. Los cargos cambiaron de denominación y están dirigidos a la promoción de servicios financieros. En la oficina está dividida el área operativa del área de negocios. Este cambio de cargos piensan que se produjeron por la integración de ambos bancos.

12. El control antes de la fusión, en parte era manual, otra parte era automatizado y estaba centralizado. Los cambios en los controles piensa un $45,5 \%$ que fue por la introducción de nuevos sistemas para medir las operaciones realizadas, minimizar las estafas y mejorar la toma de decisiones. Después de la fusión no se realizan controles manuales, todo está automatizado y sigue centralizado.

13. Antes y después de la fusión se produce la movilidad entre los puestos y piensan que no reciben ninguna contraprestación por aceptarla y en algunos casos pueden recibir viáticos. De igual manera se produce la movilidad entre las regiones y creen que se debe a políticas de la empresa, a medidas de seguridad, apoyo por estafas o por despidos del personal, reposo $o$ enfermedades.
14. Con la fusión se produjeron cambios en el salario y más del $70 \%$ de los entrevistados así lo manifestaron. Antes de la fusión estaba clasificado en un salario base, metas y pólizas y una exclusión salarial. Después de la fusión era exactamente igual lo que cambio fue el porcentaje de exclusión salaria, las metas y las pólizas.

15. También se observó que antes de la fusión le cancelaban a los empleados: horas extras, comida, transporte y otras bonificaciones. Después de la fusión no le cancelaban los tres primeros ítems y sólo hacen mención de las bonificaciones extras. Con relación a este aspecto acotaron que también la institución financiera hace uso de los premios y viajes como parte de las bonificaciones de los empleados por su puesto esto no tiene incidencia salarial en las prestaciones sociales.

16. Con la aplicación de la nueva ley el salario no tiene retroactividad y de los empleados el $44 \%$ piensan que no tiene y el otro $56 \%$ piensan que el salario si tiene retroactividad.

17. Antes de la fusión el aumento de los salarios dependía de la evaluación y de la negociación del contrato colectivo. Posterior a la fusión solo depende de la evaluación, así lo manifestó el $88,9 \%$ de los entrevistados.

18. Los elementos para la selección del personal antes de la fusión eran la experiencia en primer lugar con un $31,3 \%$ seguido de conocimientos, presencia física, pruebas y habilidades. Después de la fusión, lo fundamental es el conocimiento o nivel aca- 
démico con un $46,2 \%$ seguido de la presencia física y por último la experiencia.

19. Aun cuando no manifestaron que existe una inclinación mayor por un sexo u otro en la institución, si manifestaron que existe una tendencia de que algunos cargos estén clasificados por sexo. Por ejemplo los hombres se ubican mayormente en el área de caja y las mujeres se encuentra en el área operativa, atención al público, promotoras de servicios, en las vicepresidencias y en las gerencias medias y altas.

20. El nivel educativo exigido a los empleados también sufrió cambios ya que antes de la fusión se les exigía ser mínimo bachilleres y técnicos superiores universitarios (TSU), después de la fusión el nivel mínimo exigido es TSU y en su mayoría deben ser licenciados, ingenieros, economistas u otros. Ósea tener un titulo universitario y una mayor calificación.

21. La experiencia antes de la fusión era sumamente importante con un $88,9 \%$ ya que era interesante tener conocimiento de los procesos, por la confianza en el empleado, para compensar la falta de profesionalización y la cartera o conocimiento de los clientes; pero después de la fusión pierde importancia al ubicarse en un $44,4 \%$. La experiencia ya no es tan importante, por que lo que importa es que el empleado tenga una profesión para que reciba entrenamiento y adaptarlo a las exigencias de la institución.

22. Se determinó que el $100 \%$ de los empleados pertenecen al sindicato. Pero este es de carácter patronal existe una plancha única apoyada por la institución y las elecciones son celebradas de una manera ficticia. El 90\% manifestó que existe una contratación colectiva, de los cuales el $70 \%$ no conocen las cláusulas de este, pero si piensan que el sindicato no juega un papel importante dentro de la institución y que en la mayoría de los casos colabora con la gerencia.

23. Siempre ha existido un tipo de personal por contratación eventual y outsourcing en áreas que no tienen ingerencia con la actividad financiera antes y después de la fusión. Las áreas en las que se determinó la presencia de este personal es en: vigilancia, limpieza, anfitrionas, isbanca (INCE), departamentos administrativos, infraestructura y mantenimiento, auditoria, fideicomiso y anfitrionas.

24. Obviamente se presentaron cambios en la organización del trabajo en las oficinas con la fusión, se observaron cambios tecnológicos, una multifuncionalidad de los empleados, mayores controles y mayor responsabilidad. Los empleados piensan que los factores determinantes para que se dieran estos cambios fueron tecnológicos, por el conocimiento de los nuevos procesos y para simplificar la atención del cliente.

25. Los empleados definen la oficina bancaria antes del proceso de fusiones como centralizada, con una estructura no acorde a la modernización, manual, tradicional, pero con mayores beneficios, más cómodas, con salidas del trabajo mas temprano, funciones mas distribuidas, mas tranquilas y con una atención al cliente mas exclusiva. 
26. El $77,8 \%$ manifiestan que se produjeron cambios tecnológicos. Los cambios tecnológicos que se produjeron fueron: software, sistematización de los procesos, intranet, internet, herramientas de crédito y el home banking.

27. La edad promedio antes de la fusión manifestaron se encontraba en los 30 años y después de la fusión se encuentra aproximadamente en los 20 25 años. Esta edad de los empleados disminuyó al igual que el tiempo promedio de servicios o de antigüedad de los empleados; al ubicarse antes de la fusión entre 15 y 30 años y después de la fusión es de menos de 10 años. Con esto se puede concluir que los empleados ahora son mas jóvenes y con una carrera dentro de la institución de corta duración. Que puede estar influenciada por el incremento en la rotación del personal, ya que está más capacitado y puede ser contratado en otra institución con mejores beneficios salariales.

28. La evaluación de empleados se realiza de manera sistematizada por parte de los jefes, esta no es discutida y solo es conocida después de realizada y los elementos tomados en consideración son la evaluación de su desempeño, la atención a los clientes, la eficiencia y calidad y el cumplimiento de las metas.

29. La experiencia, el nivel académico y el conocimiento de los procesos es muy importante en los cargos medios, pero las capacidades necesarias en el negocio bancario son: que sean tomadores de decisiones, la presencia física y la aplicación de exámenes psicotécnicos y de habili- dades. Estos elementos también son importantes para los cargos gerenciales, pero están acompañados del manejo de la cartera de clientes, el conocimiento de los procesos y los años de servicio.

30. En general la opinión de los empleados en cuanto el proceso de fusiones estuvo enmarcada por lo siguiente: son traumáticas, debe haber una aceptación de los cambios, genera grandes presiones, incertidumbre y el colapso de las agencias en una primera fase. Existe una inclinación por los empleados de la institución que adquiere o predomina en la fusión; no es lo mismo comprar que ser comprados, el tiempo que duro la fusión fue bastante extendido y se produjo una inestabilidad laboral.

\section{Conclusiones}

Si bien las primeras fusiones en $\mathrm{Ve}$ nezuela a partir de 1994 incluyeron instituciones con problemas financieros; las actuales incluyen instituciones sanas con una estrategia principal de diversificación y crecimiento geográfico, por productos, por nuevas tecnologías o por una combinación entre las nombradas anteriormente; para capar nuevos nichos de mercado regionales, nacionales o internacionales.

En resumen las características de las relaciones laborales de Banesco Banco Universal a partir de las fusiones que experimentó, se pueden enumerar a continuación:

1. Reducción de las agencias y oficinas tradicionales.

2. Reducción masiva de personal o despidos colectivos o negociados, dada la aplicación de nuevas tecnologías. 
3. Incremento de la rotación de personal

4. Incremento de la movilidad entre puestos y agencias.

5. Disminución de la seguridad del empleo.

6. Incremento de la calificación y especialización del personal.

7. Eliminación de puestos de trabajo como secretarias, pruebistas y la aparición del ejecutivo vendedor o vendedor bancario.

8. Incremento de los controles de tiempo y movimiento a través del quick matic o tiempo en la cola.

9. Aparición de nuevos canales de venta a través de la Internet, home banking o banca online.

10. Inseguridad en el empleo a través de la subcontratación.

11. Alto porcentaje del paquete anual es variable y depende del cumplimiento de las metas o de las ventas cruzadas de productos.

12. Pérdida de negociación del sindicato bancario al convertirse en patronal.

13. Contratación de personal con altos niveles de calificación adaptados al puesto con un perfil determinado del cargo, con líneas de negocios preestablecidas, mensual, trimestral o semestralmente y revisables.

14. Incremento de la jornada laboral al llevarse el trabajo a casa.

15. Polifuncionalidad de los puestos de trabajo.

16. Introducción de nuevas tecnologías que reorganizan los puestos de trabajo, estableciendo una nueva organización del trabajo en las oficinas.

17. Implementación de la evaluación del desempeño.
18. Incremento del stress como una enfermedad ocupacional.

19. Choque entre las culturas de las dos instituciones a fusionarse; prevaleciendo la de la institución líder o compradora en la fusión.

20. Racionalización de las funciones y esfuerzos en los puestos de trabajo.

21. Nuevas figuras de atención al cliente: telefónica, internet, buzón de depósitos y pagos, entrega de chequeras, entre otros.

22. Concentración del poder en el mercado y reducción de la competencia dándose la creación de los megabancos.

\section{Referencias Bibliográficas}

Aguirre, Ernesto (1998). "Reformas Básicas de los Sistemas Bancarios en América Latina. Análisis, tendencias y Perspectivas" Compilado por Norton, Joseph y Aguirre, Ernesto. En: Sistemas Bancarios Latinoamericanos, reformas recientes y perspectivas. 3-32. Banco Central de Venezuela. Sistema Económico Latinoamericano (SELA) y la Agencia Española de Cooperación Internacional (AECI). Caracas-Venezuela.

Armas, Virgilio y Ugarte, Yasibyt (2001). La Banca Venezolana En Su Laberinto. El balance de la banca. Como los bancos Venezolanos sortean la crisis económica. Volumen VII. Número 1. Julio-Septiembre. CaracasVenezuela (IESA).

Asociación Bancaria de Venezuela (2002). Evolución Del Sistema Financiero Venezolano. Federación Latinoamericana de Bancos. XXXVI Asamblea Anual Reunión del Comité Latinoamericano de Administradores de Asocia- 
ciones Bancarias y Organismos Afines. Punta Cana, República Dominicana. Noviembre.

Banco Central de Venezuela (BCV) (2001). Las fusiones bancarias en Venezuela. Boletín Económico Informativo Mensual, Marzo. Año 6. Caracas-Venezuela.

Bouzas Ortiz, José Alfonso (1996). Bancomer: Reprivatización de la Banca y Flexibilización de las Relaciones Laborales. Colección: La estructura económica y social en México. Instituto de Investigaciones Económicas. Universidad Autónoma de México. Primero Edición. México.

Bouzas Ortiz, José Alfonso (2003). Democracia Sindical en el Sector Bancario. Colección: Reestructuración empresarial, democracia, representatividad y legitimidad sindical en México. No. IX. Plaza y Valdés, S.A. de C.V. México.

Bracho, Yajaira C.; Ariza, Martha; González, María C.; Jiménez, Edinson E. y otros (2002). Fusiones Bancarias En El Sistema Financiero Venezolano. Revista de Ciencias Sociales. Vol. VIII. No.2, Mayo-Agosto. pp 300-311. FACES-LUZ.

Dinero (2007). Informe Bancario 2007. Abril. Año. 18 No. 220. Caracas-Venezuela.

Ermida U., Oscar (1999). "Globalización y relaciones laborales". Revista Venezolana de Gerencia. Facultad de Ciencias Económicas y Sociales. LUZ. Año 4, No. 9. Maracaibo, Venezuela. Pág. 135-148.

Gamboa C., Teresa; Arellano, Madelein y Nava, Yuneska (2001). "Estrategias Empresariales: Aproximación A Una Tipología". Revista Venezolana de Ciencias Sociales. UNERMB. Vol. 5. No. 2. Pág. 132-154. Maracaibo, Venezuela.
González, María C.; Bracho, Yajaira; Jiménez, Edinson y Ariza, Martha (2002). Fusiones Bancarias en el Sistema Financiero Venezolano. XV Congreso de AFEIAL. Ponencia. Costa Rica. Noviembre.

Asociación Bancaria de Venezuela (2006). Evolución del sistema financiero Venezolano. Servicio de Análisis Económico. En http://www.asobanca.com.ve/data/archivos/1000/Presentaci\%F3n\%20Entorno\%20Julio\% 202006.ppt\#256,1,Diapositiva Agosto. Consultado el 05-02-07.

Jinkings, Nise (2002). "Los Bancarios Brasileños en la fase de la Reestructuración Capitalista Contemporánea". Trabajo publicado en Trayectorias, Revista de Ciencias Sociales de la Universidad Autónoma de Nuevo León. Año 4, No. 9, Mayo-Agosto. N.L. México. pp.78-99.

Ley General de Bancos y Otras Instituciones Financieras (2001). Caracas-Venezuela.

Oficina Internacional del Trabajo (OIT) (2001). La Incidencia en el Empleo de las Fusiones y Adquisiciones en el Sector de los Servicios Financieros y de la Banca. Informe para el debate de la reunión tripartita sobre la incidencia en el empleo de las fusiones y adquisiciones en el sector de los servicios financieros y de la banca. Ginebra.

Ruiz A., Héctor (2001). Fusiones bancarias. La fiebre de los megabancos. en www.elsur.cl/archivo/2001/agosto 2001/22agosto2001/elsur/reportajes/ actual1.htm. Fecha de consulta: 1503-03.

Superintendencia de Bancos 2002-2006. www.sudeban.org.ve. 